\title{
Reasoning strategies with rational numbers revealed by eye tracking
}

\author{
Patrick Plummer $^{1}$ - Melissa DeWolf ${ }^{1} \cdot$ Miriam Bassok $^{2}$ - Peter C. Gordon ${ }^{3}$. \\ Keith J. Holyoak ${ }^{1}$
}

Published online: 29 March 2017

(C) The Psychonomic Society, Inc. 2017

\begin{abstract}
Recent research has begun to investigate the impact of different formats for rational numbers on the processes by which people make relational judgments about quantitative relations. DeWolf, Bassok, and Holyoak (Journal of Experimental Psychology: General, 144(1), 127-150, 2015) found that accuracy on a relation identification task was highest when fractions were presented with countable sets, whereas accuracy was relatively low for all conditions where decimals were presented. However, it is unclear what processing strategies underlie these disparities in accuracy. We report an experiment that used eye-tracking methods to externalize the strategies that are evoked by different types of rational numbers for different types of quantities (discrete vs. continuous). Results showed that eye-movement behavior during the task was jointly determined by image and number format. Discrete images elicited a counting strategy for both fractions and decimals, but this strategy led to higher accuracy only for fractions. Continuous images encouraged magnitude estimation and comparison, but to a greater degree for decimals than fractions. This strategy led to decreased accuracy for both number formats. By analyzing participants' eye movements when they viewed a relational context and made decisions, we were able to obtain an externalized representation of the strategic choices evoked by different ontological types of entities and different types of rational numbers. Our findings using eye-tracking measures enable us to go beyond previous
\end{abstract}

Patrick Plummer

pplummer@ucla.edu

1 Department of Psychology, University of California, Los Angeles, 405 Hilgard Ave., Los Angeles, CA 90095, USA

2 University of Washington, Washington, USA

3 University of North Carolina, Chapel Hill, USA studies based on accuracy data alone, demonstrating that quantitative properties of images and the different formats for rational numbers jointly influence strategies that generate eye-movement behavior.

Keywords Eye movements and visual attention - Eye movements · Cognitive

\section{Processing strategies for quantitative reasoning}

A key issue in mathematical cognition involves delineating the strategies that people use when assessing magnitudes of perceived quantities. Most of this work has focused on simple tasks involving a single set of objects or comparisons between two symbolic magnitudes (e.g., choosing the larger of two whole numbers). In such situations, studies with children and adults suggest that different ontological types of quantities tend to evoke different strategies. Discrete (i.e., countable) entities afford a counting strategy to determine the cardinality of a set. In contrast, continuous entities do not readily afford counting but instead tend to evoke approximate magnitude estimation. (For discussion of the discrete versus continuous distinction, see Alibali, Bassok, Olseth, Syc, \& GoldinMeadow, 1999; Bassok \& Olseth, 1995).

In general, children and adults are fairly accurate in comparing the approximate sizes of numerical magnitudes (Booth \& Siegler, 2006; Halberda \& Feigenson, 2008; McCrink \& Wynn, 2004). Understanding of counting procedures develops later (Opfer \& Siegler, 2012). Children must first learn the number words and map them onto counting procedures (Gelman \& Gallistel, 1978; Rips, Bloomfield, \& Asmuth, 2008). Counting provides more precision, and once children have mastered it, it seems to replace estimation as the 
dominant strategy (Boyer, Levine, \& Huttenlocher, 2008; Mix, Levine, \& Huttenlocher, 1999; Wynn, 1997).

Other work has aimed to identify the strategies used in quantitative reasoning with more complex numerical symbols, such as the different notations for rational numbers (fractions and decimals; e.g., Obersteiner, Van Dooren, Van Hoof, \& Verschaffel, 2013; Schneider \& Siegler, 2010). There is evidence that performance on quantitative reasoning tasks is affected by alternative symbolic formats for numbers (e.g., DeWolf, Bassok, \& Holyoak, 2015; Lee, DeWolf, Bassok, \& Holyoak, 2016; Rapp, Bassok, DeWolf, \& Holyoak, 2015). A major theoretical debate has concerned whether fraction magnitudes are coded holistically (e.g., Schneider \& Siegler, 2010) or in a more compositional fashion based on the whole numbers that comprise the numerator and denominator (e.g., Bonato, Fabbri, Umilta, \& Zorzi, 2007).

An advantage of decimals over fractions for magnitude estimation has been shown in a variety of contexts and tasks, including magnitude comparison (DeWolf, Grounds, Bassok, \& Holyoak, 2014; Ganor-Stern, 2013) and number-line estimation (Iuculano \& Butterworth, 2011; Opfer \& DeVries, 2008). The ability to estimate with decimals appears to involve the same type of analog mental number line used with whole numbers (e.g., Dehaene \& Changeux, 1993; DeWolf et al., 2014; DeWolf et al., 2016). For fractions, magnitude estimates are far less precise and require additional mental calculation, as people must perform a rough approximation to division (DeWolf et al., 2014; Fazio, DeWolf, \& Siegler, 2016; Kallai \& Tzelgov, 2009), or else some other error-prone componential strategy (Bonato et al., 2007; Fazio et al., 2016). Fractions evoke greater neural activity in the intraparietal sulcus (an area consistently linked to quantitative processing; see Nieder \& Dehaene, 2009) than do either decimals or whole numbers (DeWolf et al., 2016)

Most work on processing of rational numbers has focused on magnitude comparisons; however, the representational properties of different number formats may also impact other tasks that require numerical reasoning. Whereas componential strategies for fractions are prone to error for magnitude judgments, they may be highly effective for reasoning about systematic relations between quantities. Fractions, because of their bipartite $(a / b)$ structure, can be easily mapped onto two-place quantitative relations between the cardinalities of sets. When the relations are defined for discrete, countable sets, the mapping between the entities and the number is especially clear (Rapp et al., 2015). Conversely, decimals have a unidimensional structure that readily represents continuous magnitudes (DeWolf et al., 2014; Rapp et al., 2015), making them (as reviewed above) much more effective than fractions in tasks requiring comparison of continuous magnitudes. These natural representational mappings, or alignments (Bassok, Chase, \& Martin, 1998), that hold between number format (fraction vs. decimal) and entity type (discrete vs. continuous) have implications for how people are able to successfully use these numbers in different reasoning contexts.

By contrast to the extra difficulty they pose in magnitudeoriented tasks, fractions appear to have an advantage over decimals in reasoning tasks based on two-place relationships (Empson, Levi, \& Carpenter, 2011; Kieren, 1976; Lee et al., 2016). DeWolf et al. (2015) found that adults performed much more accurately with fractions than decimals when asked to make judgments about relations between a number and a picture with two subsets composed of discrete entities. Because the components of a fraction (numerator and denominator) are each a whole number, a counting strategy is applicable when the subsets consist of discrete entities. It seems that adults are easily able to use the component parts of the fraction to map onto the components of two subsets. Decimals, which do not explicitly represent two quantities being related, appear to make it more difficult to translate the information extracted from a picture into a rational number.

\section{Using eye tracking to reveal strategic differences}

Evidence thus suggests that different notations for rational numbers impact reasoning, and there are theoretical reasons to hypothesize that fractions and decimals evoke different natural processing strategies for discrete and continuous quantities. What has been lacking, however, is any direct evidence of what strategies are evoked by different types of numerical symbols when they are applied to different types of quantities. Studies to date have provided evidence that differences in response accuracy exist but have not identified the processing strategies that yield these accuracy differences.

This deficit clouds the interpretation of some recent findings. For example, DeWolf et al. (2015; Rapp et al., 2015) have claimed that whereas fractions naturally align with discrete quantities, decimals naturally align with continuous quantities. But although DeWolf et al. found an accuracy advantage for fractions in a task requiring relational reasoning with discrete quantities, no parallel advantage was found for decimals with continuous quantities (rather, accuracy was low for both number formats). DeWolf et al. hypothesized that fractions may have led participants to impose measurement units on continuous images, which would be an error-prone strategy, whereas decimals led participants to use a comparison strategy, which tends to be inaccurate. Thus, the two number formats may have evoked different strategies for continuous images, but these strategies happened to be about equally (in)accurate. In contrast, for discrete images the two formats may have evoked different strategies that led to considerably higher accuracy for the fraction as compared to the decimal format. However, this hypothesis was entirely speculative, since the study provided no measure that could tap into 
participants' processing strategies other than response time, which was unreliable between subjects.

A methodology that potentially can reveal numerical strategies more directly is eye tracking. Recording patterns of fixations can provide evidence about relational processes as they occur (Gordon \& Moser, 2007). Furthermore, recent studies have used eye tracking to examine strategic effects as well as modulation of processing difficulty in comparison tasks involving rational numbers (Huber, Klein, Willmes, Nuerk, \& Moeller, 2014; Huber, Moeller, \& Nuerk, 2014; Hurst \& Cordes, 2016; Ischebeck, Weilharter, \& Körner, 2016; Obersteiner \& Tumpek 2016). The current study extends eye-tracking methods to investigate a task that requires relational reasoning, rather than magnitude comparison. Using a relational reasoning task adapted from DeWolf et al. (2015), we measured participants' eye movements while trying to identify specific relations between a number (either a fraction or decimal) and a picture (showing two subsets that were either discrete or continuous). Eye-tracking data allows us to test DeWolf et al.'s (2015) hypothesis that participants might be using two different types of strategies: counting and general magnitude estimation (where estimation of individual quantities serves as the precursor to comparison of two quantities). Counting is possible for discrete entities, enabling mapping of cardinalities onto the numerator and denominator of a fraction. For continuous entities, counting will be difficult (requiring the participant to impose imagined measurement units), or perhaps impossible. Instead, people may engage in some type of general magnitude estimation, weighing the relative sizes of the two subsets against one another. We aimed to determine whether and how quantitative strategies are modulated by symbolic notation, thereby allowing us to assess specific proposals about how number format and quantity type (depicted in an image) may jointly determine processing strategies.

The current study assessed patterns of eye-movement behavior in an effort to externalize strategies involving either counting or estimation/comparing. Importantly, eyemovement data can be used to generate a large portfolio of dependent measures based on fixation sequences, number of fixations, and gaze duration, which collectively can provide strong converging evidence to identify online processing strategies. For the current study, we aimed to capture instances of comparing and counting using mutually exclusive operational definitions for sequences of eye movements. Counting and comparing are not necessarily strictly alternative strategies (as there may be occasions where either or a combination of the two could prove advantageous); however, in the current study we assumed that on any specific fixation, participants might be engaged in counting or comparing, but not both simultaneously.

Previous studies have used eye-movement behavior to successfully distinguish counting from other strategic processes (Watson, Maylor, \& Bruce, 2007), such as subitizing (the confident and accurate discrimination of small quantities; Kaufman, Lord, Reese, \& Volkmann, 1949). Counting has been shown to require the incremental allocation of attentional focus across the spatial extent of countable units (Trick \& Pylyshyn, 1993, 1994). Furthermore, previous studies have suggested that inhibition of return may play a role in orienting attention and controlling eye-movement behavior during enumeration tasks (Klein \& MacInnes, 1999; Posner \& Cohen, 1984; Watson et al., 2007). These properties reflect the fact that accurate counting requires enumeration of each discrete entity exactly once, so that the task of distinguishing items in the set that were previously counted versus uncounted is crucial for precision. Thus, counting strategies should prompt sequences of fixations that move the eyes across the entire image (or at least some portion of it) in a uniform direction, only permitting returns to previously fixated regions after the enumeration is complete. A strategy involving enumeration of countable units on an image, or an attempt to imagine uniform divisions within a continuous mass, should increase the probability of stereotypical eye movements across the horizontal plane (presumably from left to right) when examining the image.

Counting is expected to inhibit returns to previously fixated regions. In direct contrast, comparison strategies would be expected to invite repeated returns to previously examined regions of the image. Comparison strategies should prompt sequences of fixations that alternate between distinct masses (regardless of ontological type). We therefore expected that comparison strategies would lead to higher rates of instances in which the eyes cross the boundary between two image subsets in an effort to compare their relative sizes. We assumed that sequences of fixations where multiple, consecutive eye movements cross the boundary between distinct elements suggest that the independent elements are being compared. Previous research has used similar techniques to classify sequences of fixations as evidence of magnitude comparisons (Ischebeck et al., 2016). Thus, we assumed that comparison strategies will be associated with consecutive eye movements crossing the image subset boundary, whereas counting strategies will be associated with consecutive eye movements within the same image subset, carrying the eyes in a uniform direction.

Strategies that rely on relative magnitude estimation and comparison should also act to focus attention toward the boundary between two distinct subsets, because fixation at the boundary will facilitate comparison of the two quantities. In contrast, counting discrete units (or mentally imposing countable units on a continuous mass) should have the effect of directing fixations to one subset at a time, and hence should draw fixations farther from the boundary. Accordingly, the average distance between fixations and the image subset boundary was taken as a measure of specific strategies.

By analyzing participants' eye movements when they viewed an image (a simple picture) and made decisions, we 
thus hoped to obtain an externalized representation of the strategic choices evoked by different ontological types of entities and different types of rational numbers. Because fractions present two distinct elements (rather than one), we expected that observers would spend more time viewing fractions than decimals. Based on prior studies, image type (discrete or continuous) should modulate eye movements on the image. In particular, a discrete image clearly lends itself to counting, whereas a continuous image does not; moreover, comparisons might occur more often when images contain continuous masses rather than discrete units. Finding support for these basic hypotheses regarding strategic eye-movement measures would serve as internal confirmation of the validity of our eyetracking methodology.

Our more basic aim was to test hypotheses regarding the potential impact of the format of the rational number on the chosen strategy. Specifically, we used eye tracking to test the hypotheses proposed by DeWolf et al. (2015): (1) fractions will promote use of counting, at least for discrete images, and (2) decimals will promote the use of comparison, at least for continuous images. Furthermore, to the extent that the format of the rational number determines the chosen strategy, fractions should reduce the use of estimation/comparison and promote the imposition of imagined measurement for continuous images, whereas decimals should reduce the use of counting and promote the use of estimation/comparison for discrete images. If fractions promote the use of counting, then we would expect higher rates of count fixations for images paired with fractions, irrespective of image type. If decimals promote the use of comparison, we would expect higher rates of compare fixations for images paired with decimals, regardless of image type.

\section{Method}

\section{Participants}

Forty undergraduates at the University of California, Los Angeles, participated in the experiment for course credit. This number of participants (higher than that assigned to each condition in De Wolf et al., 2015, Experiment 2) is sufficient to obtain reliable eye-tracking data (cf. Plummer, Perea, \& Rayner, 2014). All subjects had normal or corrected-tonormal vision.

\section{Materials and design}

Ninety-six items adapted from Experiment 2 of DeWolf et al. (2015) were used in the study. Each trial involved a display occupying a single screen. Each display consisted of two parts. On the left was a rational number (fraction or else decimal), and on the right was a simple picture (which we will term the image) that contained two subsets, consisting of either continuous or discretized entities (see Fig. 1). The images showed two types of ratio relations, both of which can be mapped to the structure of a fraction. A part-to-part ratio (PPR) is the relation between the sizes of the two subsets of a whole, whereas a part-to-whole ratio (PWR) is the relation between the size of one subset and the whole. Thus, each trial presented a decimal or fraction together with an image containing discrete units or continuous masses. The relationship between the number and image depicted either a PPR or PWR.

Note that a PPR is often termed simply a ratio, whereas a PWR is termed a proportion. Fractions are most typically used to represent part-to-whole relations (proportions). However, a ratio (part-to-part relation) can also be expressed as a fraction. ${ }^{1}$ Because interpreting fractions as something other than part-towhole ratios is unusual, we used the explicit terms part-part and part--whole in the instructions to participants to emphasize the critical distinction and make clear that both relationships can be represented by a rational number (a fraction or decimal), since both are a type of ratio. These two types of ratios were used in order to create a forced-choice task. Although there are also important conceptual differences between the two types, DeWolf et al. (2015) found no major performance differences between them (e.g., participants had little or no difficulty applying fractions to part-to-part ratios), and the distinction plays only a minor role in our own data analyses.

The participant's task was to decide whether the given rational number corresponded to a PPR or PWR ratio. For half of the items the correct relation was PPR and for half it was PWR. The design was a 2 (notation type: fractions vs. decimals) $\times 2$ (relation type: PPR vs. PWR) $\times 2$ (image type: continuous vs. discrete) within-subjects design. The stimuli were divided into two blocks of 48 items based on notation type. Within each block, image type and relation type were counterbalanced across items and presented in randomized order. The order of presentation for the fraction and decimal blocks was counterbalanced.

The values of fractions and decimals were always less than one. Fractions presented numerators that ranged from 1 to 11 and denominators that ranged from 4 to 16 . Decimals were always presented rounded to two decimal places. Decimals and fractions were rendered using the same font size and presented centered on left side of the screen. Fractions were approximately 200 pixels in height and decimals approximately 70 pixels in height. The images shown for fractions and decimals were matched. The two subsets of each image were

\footnotetext{
${ }^{1}$ In general, a ratio is the quotient of two values, whereas a fraction is the quotient of two whole numbers. Thus, a ratio can be written as a fraction whenever the values being related are whole numbers. According to the Encyclopedia Britannica entry for ratio, "The ratio of $a$ to $b$ can be written $a: b$ or as the fraction $a / b$ " (see https://www.britannica.com/topic/ratio).
} 


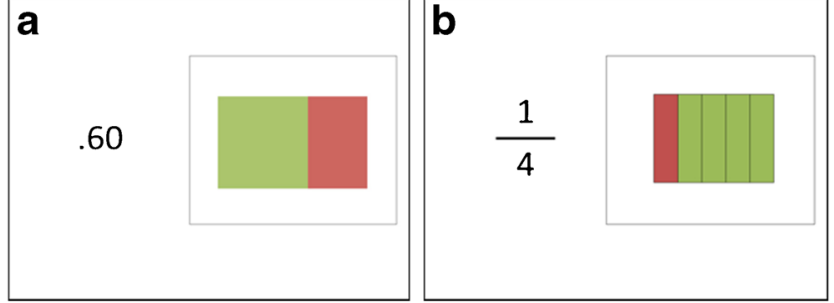

Fig. 1 Examples of displays with a decimal paired with a continuous image, and a fraction paired with a discrete image: (a) decimal with a PWR continuous image (if the image were depicting PPR the decimal value would equal 0.67); (b) fraction with a PPR discrete image (if panel if image were depicting PWR the fraction would equal 1/5)

demarcated using green and red colors (in practice items, yellow and blue colors were used). For discrete images, dark lines were used for delineation of equal-sized units within each color and each image. To ensure that the individual rectangles were clearly differentiated, the width of each rectangle was varied from 30 to 40 pixels (allowing wider rectangles when they were fewer in number). Continuous images were constructed in the same manner as discrete images with the only difference being the absence of delineation within each color region. Images varied which color appeared on the leftmost side and which color represented the larger subset. Each image was 230 pixels in height. Image width varied across items, ranging from 203 to 516 pixels in width, subtending approximately 8 to 20 degrees of visual angle $(M=395$ pixels in width). Mean width of images was 411 pixels (subtending approximately 16 degrees of visual angle) for continuous images and 378 pixels (subtending approximately 15 degrees of visual angle) for discretized images, a difference that was not statistically significant $(p=.10)$.

\section{Procedure}

Stimuli were displayed on a 21-in. ViewSonic Graphic Series G225f CRT monitor with a screen resolution of $1024 \times 768$ with subjects seated at a distance of $55 \mathrm{~cm}$ (although there was no physical mechanism for restricting head movements). Eye movements were recorded using an SR Research Eye Link II head-mounted eye tracker sampling at $250 \mathrm{~Hz}$. Question accuracy and response time were recorded on each trial. A ninepoint calibration procedure for the tracker was performed prior to each of the two blocks. The calibration was checked after each trial using a drift-correction procedure, and recalibration was performed when necessary.

The instructions stated that a number and an image would be displayed on the screen, and the task would be to identify whether the given number represented a part-part or partwhole relation in the image. Responses were made using a hand-held remote console. During instructions, participants were shown examples of the two relation types using both discrete and continuous images. Following the instructions, participants were given a practice item and told to identify the correct relation. Feedback was given (irrespective of response accuracy) as well as an explanation of why the number matched one of the two relations. The explanation also stated what the numerical value would be if the alternative relation had been matched. Participants were then given a second practice item in which the number matched the alternative relation, with the same explanation process. This feedback was intended to make it clear that numbers (whether decimal or fraction) were being used to depict both part-to-part ratios and part-to-whole ratios. The notation type of the rational numbers used in the instructions and practice items corresponded to the notation type presented in the subsequent block of items. Participants received instructions and practice items prior to eye-tracker calibration before each block. Only after the participant confirmed their understanding of the task (including the distinction between the two alternative ratio types) did the experimenter calibrate the tracker and initiate the first trial of the block.

Each test trial began with the participant holding their gaze on a fixation point near the left edge of the screen. At the start of the trial, the number would appear to the right of the fixation point (on the left half of the screen). Once the number had been displayed for $1,000 \mathrm{~ms}$, the image appeared to the right of the number. The latency in presenting the image was intended to give participants sufficient time to encode the number prior to engaging with the image (regardless of symbolic notation). Once displayed together, the number and the image remained on the screen until the participant selected one of the two relation types as the match, ending the trial. Participants were allowed 25 seconds to respond after the onset of the image (although no participant required the maximal time on any trial). Participants were not encouraged to answer as quickly as possible; rather, the experimenter stressed accuracy during the instructions and training block. Participants completed 96 test trials in total (12 for each of the eight experimental conditions across the two blocks).

\section{Results}

\section{Eye tracking: local analyses}

In order to test hypotheses regarding counting and estimation/ comparison strategies, we performed a series of analyses that focused on specific, local characteristics of viewing patterns (i.e., sequences of consecutive fixations) on the image. Sequences were classified into distinct categories intended to characterize counting or comparing. Any sequence of three consecutive fixations within the same subset of the image where the eyes moved in a rightward direction between each fixation was interpreted as an instance of counting. The third fixation in such a sequence would be indexed as a count 
fixation; additionally, each additional fixation within the same subset of the image following a rightward eye movement was also indexed as a count fixation (until the eyes moved to the left, to the other subset, or off the image). This classification criterion implies that a sequence of five consecutive fixations within the same subset would result in three count fixations (i.e., each fixation to the right of the initial count fixation was coded as a count fixation).

Comparing behavior was also assessed based on fixations on the image within each trial. Any sequence of three consecutive fixations where the boundary between image subsets (i.e., the two colors) was crossed twice was interpreted as a comparison. The third fixation in such a sequence was classified as a compare fixation. Each subsequent fixation resulting from an eye movement between subsets was also classified as a compare fixation (until the participant made two consecutive fixations within one subset or fixated anywhere outside the image). Note that the count and compare measures are operationalized as sequences of fixations that are mutually exclusive (i.e., no fixation could be simultaneously classified as a both). The measures are also conservative in that a threefixation sequence is required before a classification is made.

To supplement the measures that capture specific sequences of eye movements on the image, we also assessed measures that were based on all fixations on the image, which capture broader aspects of attention allocation and processing difficulty. The measure image fixations, defined as the sum of the total fixations on the image, was interpreted as a measure of overall attention (i.e., a proxy for where processing resources were focused). Boundary distance - the mean distance between each image fixation (measured horizontally in pixels) and the demarcation between the two colored subsets of the imagewas interpreted as a measure reflecting attention allocation within the image. Closer mean boundary distance should reflect simultaneous allocation of attention to both subsets, and hence supports a comparison strategy; whereas farther boundary distance should reflect attention to one subset at a time, which is consistent with counting (perhaps without eye movements). The boundary distance measure thus may reveal covert shifts in attention (i.e., changes in the location of attention without a corresponding eye movement; Posner, 1980) that might accompany processing of a subset (farther distance) or else comparison (closer distance).

Eye movements on the region of the screen where the number appeared were analyzed with regard to number format and image type. The measure number fixations reflects the total fixations on the number for an entire trial. The measure number returns indicates the total fixations on the number that immediately follow a fixation on the image. Returning from the image to reexamine the number is indicative of additional processing of the number that occurred once the complete relational context was available (i.e., once the number and image had both been seen).
Any fixations on the image area that ended prior to the presentation of the image were removed before analysis. This selection criterion resulted in the loss of $1.2 \%$ of all data (511 fixations). Mean question accuracy was above chance for all experimental conditions. All dependent measures were analyzed using a 2 (number format: decimal vs. fraction) $\times 2$ (image type: continuous vs. discrete) $\times 2$ (relation type: PPR vs. PWR) within-subjects ANOVA.

\section{Accuracy}

Table 1 shows mean relation-identification accuracy across number format and image type. The main effect of number format was not significant; however, there was a main effect of image type, $F(1,39)=5.93, M S E=.042, p=.02, \eta_{p}^{2}=.132$, with accuracy higher overall for discrete images when compared to continuous images. There was also a significant interaction of number format and image type, $F(1,39)=13.13$, $M S E=.157, p<.01, \eta_{p}^{2}=.252$, revealing that accuracy was highest for discrete images matched with fraction notation, with no differences in accuracy across the other three conditions. This interactive pattern of image and number format replicates that reported by DeWolf et al. (2015). Accuracy was $77 \%$ and $70 \%$ for PWR and PPR items, respectively, a difference that was reliable, $F(1,39)=15.29, M S E=.445, p<$ $.01, \eta_{p}^{2}=.282$. As there were no significant two- or three-way interactions involving relation type, and the effect of relation type is not of particular theoretical interest, all subsequent measures were analyzed after collapsing across PWR and PPR items.

\section{Eye movements on the image}

The measures of count fixations and compare fixations were intended to capture the extent to which strategic processing differences were associated with differences in image and number formats. Figure 2 depicts count fixations and compare fixations across the number and image types (also summarized in Table 1). A main effect of image type was obtained, with discrete images eliciting more count fixations than did continuous images, $F(1,39)=22.83, M S E=17.51, p$ $<.01, \eta_{p}^{2}=.369$. This finding was of course expected, and serves as confirmation of the validity of our eye-tracking measure of counting behavior.

In addition, a significant correlation was obtained between the observed number of count fixations and the number of countable units contained within the image on each trial, $r=$ $0.194, p<.01$, whereas the observed number of compare fixations showed essentially no correlation with the number of countable units $(r=-.004)$. There was no significant effect of number format on count fixations, and no significant interaction of number format and image type. 
Table 1 Means (standard errors) for all measures across conditions

\begin{tabular}{cllll}
\hline & Fraction - Discrete & Fraction - Continuous & Decimal - Discrete & Decimal - Continuous \\
\hline $\begin{array}{c}\text { Accuracy } \\
\text { Image measures }\end{array}$ & $0.79(.03)$ & $0.72(.03)$ & $0.72(.03)$ & $0.72(.03)$ \\
Count fixations & $1.08(0.19)$ & $0.37(0.06)$ & $0.94(0.17)$ & $0.33(0.05)$ \\
Compare fixations & $0.45(0.05)$ & $0.57(0.06)$ & $0.64(0.07)$ & $0.66(0.08)$ \\
Boundary distance & $80.3(1.6)$ & $71.6(2.2)$ & $75.1(2.1)$ & $68.8(2.2)$ \\
Image fixations & $7.01(0.58)$ & $6.60(0.58)$ & $8.05(0.79)$ & $6.37(0.52)$ \\
Number measures & & & & $2.98(0.16)$ \\
Number fixations & $3.54(0.17)$ & $4.03(0.21)$ & $0.61(0.07)$ & $0.56(0.07)$ \\
Number returns & $0.56(0.06)$ & $0.69(0.07)$ & & \\
\hline
\end{tabular}

The overall frequency of count fixations could have been influenced by differences in the total number of fixations (and the total amount of time spent) on the image across conditions. Previous studies have addressed this potential confound by using measures based on fixation proportions rather than raw fixation frequency (Huber et al., 2014; Obersteiner \& Tumpek, 2016). Critically, when the measure of counting was computed as the proportion of fixations on the image classified as count fixations for each trial, the pattern of significant effects across factors did not differ from those obtained using the measure based on simple count fixation frequency. Discrete images yielded higher proportions of fixations classified as count fixations when compared to continuous images (with count fixation proportions averaging 0.113 and 0.054 for discrete and continuous images, respectively. Furthermore, the proportion of count fixations for fraction items $(M=0.089)$ did not significantly differ from the

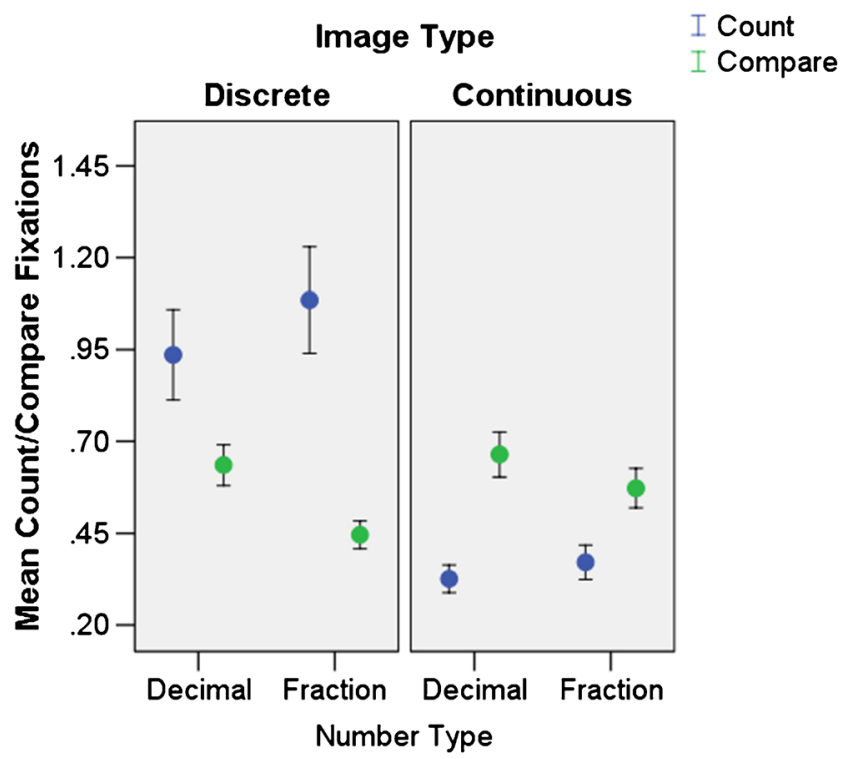

Fig. 2 Count and compare fixations across conditions. The $y$-axis shows the mean number of count and compare fixations per trial, broken down by number formats and image types, with standard error bars proportion for decimal items $(M=0.078)$. Discrete images thus evoked counting about equally across decimal and fraction notations.

A very different pattern was observed for compare fixations. A greater number of compare fixations was made when the image was continuous rather than discrete, $F(1$, 39) $=6.94, M S E=0.24, p=.01, \eta_{p}^{2}=.151$. There was also a main effect of number format, with a higher frequency of compare fixations on the image when the number was presented in decimal rather than fraction format, $F(1,39)=$ 5.57, $M S E=0.78, p=.02, \eta_{p}^{2}=.125$. The interaction of image type and number format on frequency of compare fixations did not reach significance. As in the analyses of count fixations, when the measure of comparing was computed as the proportion of fixations on the image region classified as compare fixations for each trial, the pattern of significant effects was identical to the results using raw frequency of compare fixations. Continuous images yielded a higher proportion of compare fixations than did discrete images (0.075 and 0.063 , respectively), and decimals yielded a higher proportion of compare fixations than did fractions (0.076 and 0.063, respectively), with no significant interactions. Observers thus compared the two subsets of the image more often when it showed continuous masses and when the number was presented as a decimal.

Similar analyses were performed for the more general measures of fixation patterns on the image. Discrete images received significantly more total fixations than continuous images, $F(1,39)=18.48, M S E=43.88, p<.01, \eta_{p}^{2}=.321$. There was no reliable main effect of number format, but there was a significant interaction of number and image type, revealing that the increase in image fixations for discrete images was only obtained when paired with decimals, $F(1,39)=6.02$, $M S E=16.07, p=.02, \eta_{p}^{2}=.134$. Total time spent viewing the image, and overall response time, yielded the same interactive pattern between number format and image type (both $p$ s $<.05)$. These results demonstrate that the format of the 
number and image type jointly influenced processing of the image during the task, with decimals paired with discrete images creating the highest cognitive load (as shown by the increase in response time as well as total fixations and viewing time on the image).

Mean boundary distances for all fixations on the image, across image and number formats, are shown on Table 1 . Continuous images resulted in fixations being closer to the boundary between subsets, as compared to discrete images, $F(1,39)=33.71, M S E=2245.55, p<.01, \eta_{p}^{2}=.464$. In addition, decimal notation resulted in fixations being closer to the boundary between the two subsets in the image when compared to fraction notation, $F(1,39)=7.16, M S E=655.52$, $p=.01, \eta_{p}^{2}=.155$. There was no reliable interaction between number format and image type for boundary distance. The results show that both image and number format influence the location of fixations when viewing the image (i.e., the focus of attention). Fractions and discrete images direct attention away from the subset boundary whereas decimals and continuous images direct attention toward image subset boundary.

\section{Eye movements on the number}

Given the larger spatial extent of fraction as compared to decimal notation, and the bipartite format of fractions as compared to the one-dimensional format of decimals, it was expected that fractions would receive longer viewing times than decimals. Indeed, fractions received more fixations than decimals, $F(1,39)=51.11, M S E=30.58, p<$ $.01, \eta_{p}^{2}=.567$. There was also a main effect of image type, with numbers receiving more fixations when images displayed continuous masses as compared to discretized units, $F(1,39)=4.66, M S E=1.20, p<.01, \eta_{p}^{2}=.107$. In addition, there was a significant interaction between number format and image type for this measure, $F(1,39)=$ 15.18, MSE $=4.06, p<.01, \eta_{p}^{2}=.280$. As shown in Table 1, the increase in fixations on the number with continuous images relative to discrete images was only observed for fractions. There was no substantial difference between image types when numbers were in decimal notation (in fact, the trend was in the opposite direction).

A similar interaction between number format and image type was observed for number returns, $F(1,39)=9.03, M S E$ $=0.30, \mathrm{p}<.01, \eta_{p}^{2}=.188$. Participants returned to examine the number more often when presented with continuous as compared to discrete images, but only when fraction format was used. Neither the main effect of number format nor of image type reached significance for number returns. Because number format and image type interacted for both measures of looking at the number, differences in eye movements on the number cannot be solely due to fractions covering a larger spatial extent or having a more complex structure than decimals.

\section{Eye tracking: global scan-path analysis}

The findings presented above indicate that number format systematically influences strategic choices, as evidenced by differences in observed viewing patterns. In order to further explore the effect of number format on strategic choices, a more global analysis was conducted to examine scan-path similarity across conditions for each participant. This analysis employed the Needleman-Wunsch algorithm implemented in the ScanMatch program (Cristino, Mathôt, Theeuwes, \& Gilchrist, 2010) using MATLAB and the bioinformatics toolbox. The algorithm was used to compute scores that reflect the overall similarity between the sequences of fixations associated with each trial. First, the sequence of fixations for each trial was converted to a representation of the spatial (i.e., fixation locations) and temporal (fixation durations) aspects of the particular eye-movement pattern (i.e., scan path). For the analysis, the entire display screen $(1024 \times 768$ pixels $)$ for each trial was divided into a $12 \times 8$ grid of spatial bins. Fixations were binned spatially using the $12 \times 8$ grid and binned temporally based on temporal bins of $100 \mathrm{~ms}$.

Next, each scan path was compared with the scan path of every other trial within the same condition (determined by number format and image type), creating a $24 \times 24$ item similarity score matrix. Similarity scores could vary from zero, indicating no similarity whatsoever, to one, indicating perfect similarity. Self-comparisons, which inevitably yield perfect similarity scores of one, were removed from the analysis. In addition, fraction trials were compared with decimal trials for each of the two image types, creating two additional $24 \times 24$ matrices that could be used to compare scan-path similarity across the two number formats. Thus, in total, six sets of similarity matrices were constructed for each participant. These matrices assessed the similarity of fraction trials to other fraction trials (fraction-to-fraction), decimal trials to other decimal trials (decimal-to-decimal), and fraction trials to decimal trials (fraction-to-decimal), separately for discrete and continuous images. Comparisons between the former two conditions versus the third serve to assess whether or not similarity of scan paths was greater for trials of the same versus different number format (holding image type constant).

The specific strategic measures (e.g., count fixations) as well as the broader eye-movement measures (e.g., image fixations) permitted analyses of local eye-movement behavior on the image and the number. Scan-path similarity scores, which serve to complement these measures of local processing, would be expected to reflect systematic regularities in global viewing behavior across conditions in a holistic fashion. If number format influences strategic choices, independent of the image format, then scan paths for items sharing the same number format should be more similar to each other than to items from the same image condition but the alternative number format. If so, then fraction-to-fraction as well as decimal- 
to-decimal scan-path similarities should be higher than fraction-to-decimal scan-path similarity. In contrast, if one or both of the number formats is not a strong determinant of eyemovement behavior during the reasoning task, then similarity of scan paths would not differ for trials of the same versus different number formats.

The results of the global analyses of scan-path similarity indicated that the impact of number format differed between the two image types. For items with discrete images, decimalto-decimal similarity $(M=0.483, S D=0.06)$ and fraction-tofraction similarity $(M=0.484, S D=0.05)$ were both significantly higher than fraction-to-decimal similarity $(M=0.446$, $S D=0.06), t(78)=2.70, p<.01$ and $t(78)=2.99, p<.01$, respectively. For items with continuous images, decimal-todecimal similarity $(M=0.496, S D=0.05)$ but not fraction-tofraction similarity $(M=0.467, S D=0.06)$ was reliably higher than fraction-to-decimal similarity $(M=0.451, S D=0.06)$, $t(78)=3.66, p<.01$ and $t(78)=1.29$, respectively.

These results fit well with the findings based on local measures of eye-movement behavior. It appears that decimals, more so than fractions, prompt strategic choices that hold regardless of the type of image being displayed. We saw earlier that decimals evoke relatively high rates of comparison, which could be driving scan-path similarity. In general, fractions paired with discrete images afford the easiest and most precise strategy (counting). This relative uniformity of strategy may have contributed to high fraction-to-fraction similarity for items with discrete images. In contrast, fractions paired with continuous images may have elicited more variable strategies, and hence more variable scan paths.

\section{Discussion}

By analyzing eye movements during a relation identification task, we were able to obtain an externalized representation of the strategic choices evoked by different ontological types of entities and different types of rational numbers. Whereas previous work (DeWolf et al., 2015) was limited to response accuracy as the primary dependent measure, we were able to take advantage of an extensive portfolio of measures derived from eye-tracking data. These measures provide strong converging evidence that specific processing strategies yield the observed differences in response accuracy across conditions.

Multiple types of eye-movement measures were employed (see Table 1). First, count fixations and compare fixations provided direct indicators of counting and comparison strategies, respectively, based on specific sequential eye-movement patterns. Second, boundary distance provided a supplemental indicator of strategy use, with fixations close to the boundary between subsets facilitating comparisons and fixations far from the boundary facilitating processing of a single subset (perhaps by counting even in the absence of eye movements).
Third, image fixations indexed overall attention to the image, which likely reflects the cognitive load imposed by processing the perceptual quantities. Fourth, number fixations and number returns indexed attention to the numerical symbol (overall, and subsequent to viewing the image, respectively). These measures likely reflect the cognitive load imposed by mapping perceived quantities to the numerical symbol. Count fixations, compare fixations, and boundary distance served as markers of specific strategies, whereas image fixations, number fixations, and number returns were measures of overall processing difficulty. While there were clear collinearities in the set of measures employed, the multiple variables served to illuminate distinct aspects of element-specific processing during the relation identification task. Finally, a global measure of scan-path similarity was employed to supplement the analyses based on local eye-movement measures, providing additional evidence concerning the impact of number format on eyemovement behavior.

With respect to response accuracy, we replicated the pattern obtained by DeWolf et al. (2015): Accuracy was highest when fractions were paired with discrete images and about equally low for the other three conditions. The converging evidence provided by this portfolio of eye-tracking measures makes it possible to identify the processing strategies that lead to differential accuracy, by providing detailed insights into the online strategies people use to map rational numbers onto relations between perceptual quantities. Eye-tracking measures allow us to delineate the unique pattern of preferred strategies underlying reasoning performance for each combination of number format and image type.

Across participants and items there were doubtless cases in which strategic measures were indexed for trials on which the indicated strategy was not actually being performed. On the other hand, there were also certainly trials in which enumeration of at least one set of discrete items could be accomplished through subitizing, or trials in which comparisons were accomplished without overt shifts of attention to the image (i.e., cases where no strategic fixations would be indexed). Furthermore, participants were free to employ idiosyncratic strategies for determining the correct alternative in the task, making use of a variety of visual and conceptual cues.

Nonetheless, we believe the strategic measures were sufficiently conservative to warrant strong interpretation of reliable differences. Discrete images elicited a greater frequency of count fixations, a lesser frequency of compare fixations, and a higher average boundary distance, relative to continuous images. In sharp contrast, continuous images elicited less counting and more compare fixations, and lower average boundary distance, relative to discrete images. These findings further confirm that ontologically different entities promote distinct processing strategies. More specifically, the results show that during a relational reasoning task, continuous images prompted comparison and discrete images prompted counting. 
Perhaps of greater theoretical interest, the findings revealed that strategic processing also varied as a function of rational number format. Decimal format prompted higher rates of comparing across both image types; in contrast, fractions did not appear to prompt counting independent of image type. Furthermore, decimal format prompted fixations on the image that tended to be closer to the boundary between image subsets, whereas fractions prompted fixations that tended to be farther from the boundary. These differences might reflect strategic differences in the allocation of attention across the image. The global scan-path analysis further indicated that decimal format yielded some regularity in the patterns of eye movements over and above the regularity imposed by image type.

With respect to cases in which number format and image format were aligned, our findings lend support to our entering hypotheses. The case in which a fraction is paired with a discrete image provides a natural semantic alignment (DeWolf et al., 2015; Rapp et al., 2015), because precise counts of the subsets can be mapped directly onto the numerator and denominator of the fraction. This favorable case, which yields the highest accuracy, elicited the greatest frequency (and proportion) of count fixations and the lowest rate of compare fixations, coupled with maximal boundary distance. The eye-tracking results suggest that participants were better able to determine the correct relation by mapping the cardinalities of the two distinct sets in the image to the two distinct elements of the fraction. Whereas componential processing of fractions in terms of their constituent whole numbers is not reliable for tasks that require magnitude processing (Schneider \& Siegler, 2010), a componential approach is highly effective in the relation identification task.

In contrast, when a decimal was paired with a continuous image, this natural alignment did not afford increases in precision or accuracy, because participants must rely on magnitude estimation and comparison strategies, which are inherently less precise than a counting strategy. Cases of this type elicited the greatest frequency (and proportion) of compare fixations and the lowest rate of count fixations, coupled with minimal boundary distance. Overall, the eye-tracking measures point to a strong preference for a counting strategy when fractions are mapped onto discrete images and a strong preference for an estimation/comparison strategy when decimals are mapped onto continuous images, supporting two of the predictions of DeWolf et al. (2015).

More surprisingly, and contrary to what DeWolf et al. (2015) had predicted based on accuracy data alone, counting was typical for discrete images even when these were paired with decimals. A discrete image apparently evokes counting even for a number format (decimals) that does not align well with a counting strategy, leading to lower response accuracy for trials with decimals than fractions. This finding is particularly striking because adults should easily be able to ignore the segments and view the images as two continuous subsections, given that the similar colored elements are grouped together. The fact that discrete images favored counting not only for fractions but also for decimals is consistent with evidence (e.g., Boyer et al., 2008) that older children and adults are strongly predisposed to count whenever possible, whether or not counting is the optimal strategy. Counting does not align naturally with decimals, and estimation is less accurate than counting; hence, neither strategy is particularly well-suited for evaluating relations when decimals are paired with discrete images. Moreover, interactions observed in total fixations on the image, total time viewing the image, and overall response time all indicated that this misaligned condition imposed a particular type of cognitive load, prompting more attention allocation to the image than in any other condition. Apparently, despite having counted the discrete units, in this condition participants ultimately relied on comparison strategies to produce a response.

When a fraction was paired with a continuous image, the results offered little support for DeWolf et al.'s (2015) hypothesis that participants attempt to impose discrete units onto the image. However, there was some evidence that fractions directed attention away from the boundary between image subsets. The condition in which a fraction was paired with a continuous image yielded the most fixations on the number, and the highest frequency of returns to the number after viewing the image in relation, relative to the three other conditions based on combinations of number format and image type. The results suggest that the cognitive load imposed by the specific misalignment of fractions and continuous images drove participants' attention toward the number, perhaps in an attempt to convert the two-dimensional fraction into a more basic representation of magnitude.

It is interesting that while the two misaligned conditions both showed evidence of increased processing load, the nature of the extra processing differed. Relatively high rates of count and compare fixations were observed when a decimal was paired with a discrete image. Although no interactions based on count or compare fixations reached significance, the extra attention (i.e., fixations and time) was revealed by the interaction in image fixations. Presumably, attempts to coerce the decimal representation into a fraction (to correspond more easily with the countable sets) would have directed more attention to the number (as reflected in number fixation and number returns); however, the results showed no indications of such a strategy. Conversely, when a fraction was paired with a continuous image, extra fixations were directed at the number (as shown by the interactions in number fixations and number returns). It appears that in the former case people have more difficulty extracting magnitudes from the image, whereas in the latter case people have more difficulty relating the magnitudes to the two-dimensional form of the fraction. Contrary to a hypothesis raised by DeWolf et al. (2015), in 
the latter case we found no compelling evidence that people attempted to mentally impose discrete units on continuous images, even when paired with fractions.

The present findings using eye tracking thus support some, but not all, of our entering hypotheses regarding the relation identification task. DeWolf et al.'s (2015) hypothesis that different strategies are used for continuous images depending on number format (even though these strategy differences do not translate into accuracy differences) was supported directly by the effects observed on strategic measures as well as measures that reflected processing difficulty and the allocation of attention. Collectively, the results of the current study demonstrate that rational numbers using distinct symbolic notations differ in their coherence with a relational context that requires quantitative reasoning about perceived quantities. Fractions naturally align with countable sets, whereas decimals align with continuous masses. Converging evidence from a portfolio of eye-tracking measures revealed online differences in strategic processing that stem from the different procedural affordances of alternative number formats coupled with distinct ontological quantity types.

Acknowledgements P. Plummer was supported by a University of California President's Postdoctoral Fellowship during the completion of this work, and M. DeWolf was supported by NSF Fellowship DGE1144087.

Author contributions All authors contributed to the planning of the study design and analysis. P. Plummer, M. DeWolf, P. C. Gordon, and K. J. Holyoak contributed to the drafting of the manuscript. P. Plummer, M. DeWolf, and M. Bassok constructed the stimuli for the study. P. Plummer was responsible for data collection. P. Plummer, M. DeWolf, and P. C. Gordon performed all data analysis for the study.

\section{References}

Alibali, M. W., Bassok, M., Olseth, K. L., Syc, S., \& Goldin-Meadow, S. (1999). Illuminating mental representations through speech and gesture. Psychological Science, 10, 327-333.

Bassok, M., Chase, V. M., \& Martin, S. A. (1998). Adding apples and oranges: Alignment of semantic and formal knowledge. Cognitive Psychology, 35, 99-134. doi:10.1006/cogp.1998.0675

Bassok, M., \& Olseth, K. L. (1995). Object-based representations: Transfer between cases of discrete and continuous models of change. Journal of Experimental Psychology: Learning, Memory, and Cognition, 21, 1522-1538.

Bonato, M., Fabbri, S., Umilta, C., \& Zorzi, M. (2007). The mental representation of numerical fractions: Real or integer? Journal of Experimental Psychology: Human Perception and Performance, 33, 1410-1419.

Booth, J., \& Siegler, R. (2006). Developmental and individual differences in pure numerical estimation. Developmental Psychology, 41(6), 189-201.

Boyer, T. W., Levine, S. C., \& Huttenlocher, J. (2008). Development of proportional reasoning: Where young children go wrong. Developmental Psychology, 44, 1478-1490.
Cristino, F., Mathôt, S., Theeuwes, J., \& Gilchrist, I. D. (2010). ScanMatch: A novel method for comparing fixation sequences. Behavior Research Methods, 42(3), 692-700.

Dehaene, S., \& Changeux, J.-P. (1993). Development of elementary numerical abilities: A neuronal model. Journal of Cognitive Neuroscience, 5, 390-407.

DeWolf, M., Bassok, M., \& Holyoak, K. J. (2015). Conceptual structure and the procedural affordances of rational numbers: Relational reasoning with fractions and decimals. Journal of Experimental Psychology: General, 144(1), 127-150.

DeWolf, M., Chiang, J. N., Bassok, M., Holyoak, K. J., \& Monti, M. M. (2016). Neural representations of magnitude for natural and rational numbers. NeuroImage. doi:10.1016/j.neuroimage.2016.07.052

DeWolf, M., Grounds, M. A., Bassok, M., \& Holyoak, K. J. (2014). Representation and comparison of magnitudes for different types of rational numbers. Journal of Experimental Psychology: Human Perception and Performance, 40(1), 71-82.

Empson, S. B., Levi, L., \& Carpenter, T. P. (2011). The algebraic nature of fractions: Developing relational thinking in elementary school. In J. Cai \& E. Knuth (Eds.), Early algebraization: A global dialogue from multiple perspectives (pp. 409-428). Berlin: Springer.

Fazio, L. K., DeWolf, M., \& Siegler, R. S. (2016). Strategy use and strategy choice in fraction magnitude comparison. Journal of Experimental Psychology: Learning, Memory, and Cognition, 42(1), 11-16.

Ganor-Stern, D. (2013). Are $1 / 2$ and 0.5 represented in the same way? Acta Psychologica, 142(3), 299-307.

Gelman, R., \& Gallistel, C. R. (1978). The child's understanding of number. Cambridge: Harvard University Press.

Gordon, P. C., \& Moser, S. (2007). Insight into analogy: Evidence from eye movements. Visual Cognition, 15, 20-35.

Halberda, J., \& Feigenson, L. (2008). Developmental change in the acuity of the "Number sense": The approximate number system in 3-, 4-, 5-, and 6-year-olds and adults. Developmental Psychology, 44, $1457-1465$.

Huber, S., Klein, E., Willmes, K., Nuerk, H. C., \& Moeller, K. (2014). Decimal fraction representations are not distinct from natural number representations-Evidence from a combined eye-tracking and computational modeling approach. Frontiers in Human Neuroscience, 8, 172-186.

Huber, S., Moeller, K., \& Nuerk, H. C. (2014). Adaptive processing of fractions-Evidence from eye-tracking. Acta Psychologica, 148, $37-48$.

Hurst, M., \& Cordes, S. (2016). Rational-number comparison across notation: Fractions, decimals, and whole numbers. Journal of Experimental Psychology: Human Perception and Performance, 42(2), 281-293.

Ischebeck, A., Weilharter, M., \& Körner, C. (2016). Eye movements reflect and shape strategies in fraction comparison. Quarterly Journal of Experimental Psychology, 69(4), 713-727.

Iuculano, T., \& Butterworth, B. (2011). Understanding the real value of fractions and decimals. Quarterly Journal of Experimental Psychology, 64(11), 2088-2098.

Kallai, A. Y., \& Tzelgov, J. (2009). A generalized fraction: An entity smaller than one on the mental number line. Journal of Experimental Psychology: Human Perception and Performance, $35,1845-1864$.

Kieren, T. E. (1976). On the mathematical, cognitive, and instructional foundations of rational number. In R. Lesh (Ed.), Number and measurement: Papers from a research workshop (pp. 101-144). Columbus: ERIC/SMEAC.

Lee, H. S., DeWolf, M., Bassok, M., \& Holyoak, K. J. (2016). Conceptual and procedural distinctions between fractions and decimals: A crossnational comparison. Cognition, 147, 57-69.

McCrink, K., \& Wynn, K. (2004). Large-number addition and subtraction by 9-month-old infants. Psychological Science, 15, 776-781. 
Mix, K. S., Levine, S. C., \& Huttenlocher, J. (1999). Early fraction calculation ability. Developmental Psychology, 35, 164-174.

Nieder, A., \& Dehaene, S. (2009). Representation of number in the brain. Annual Review of Neuroscience, 32, 185-208.

Obersteiner, A., \& Tumpek, C. (2016). Measuring fraction comparison strategies with eye-tracking. ZDM Mathematics Education, 48, 255-266. doi:10.1007/s11858-015-0742-Z

Obersteiner, A., Van Dooren, W., Van Hoof, J., \& Verschaffel, L. (2013). The natural number bias and magnitude representation in fraction comparison by expert mathematicians. Learning and Instruction, 28, 64-72. doi:10.1016/j.learninstruc.2013.05.003

Opfer, J. E., \& DeVries, J. M. (2008). Representational change and magnitude estimation: Why young children can make more accurate salary comparisons than adults. Cognition, 108(3), 843-849.

Opfer, J. E., \& Siegler, R. S. (2012). Development of quantitative thinking. In K. J. Holyoak \& R. G. Morrison (Eds.), Oxford handbook of thinking and reasoning (pp. 585-605). New York: Oxford University Press.

Plummer, P., Perea, M., \& Rayner, K. (2014). The influence of contextual diversity on eye movements in reading. Journal of Experimental Psychology: Learning, Memory, and Cognition, 40(1), 275-283.

Posner, M. I. (1980). Orienting of attention. Quarterly Journal of Experimental Psychology, 32, 3-25.
Rapp, M., Bassok, M., DeWolf, M., \& Holyoak, K. J. (2015). Modeling discrete and continuous entities with fractions and decimals. Journal of Experimental Psychology: Applied, 21(1), 47-56.

Rips, L. J., Bloomfield, A., \& Asmuth, J. (2008). From numerical concepts to concepts of number. Behavioral and Brain Sciences, 31, 623-642.

Schneider, M., \& Siegler, R. S. (2010). Representations of the magnitudes of fractions. Journal of Experimental Psychology: Human Perception and Performance, 36, 1227-1238. doi:10.1037/ a0018170

Trick, L. M., \& Pylyshyn, Z. W. (1993). What enumeration studies can show us about spatial attention: Evidence for limited capacity preattentive processing. Journal of Experimental Psychology: Human Perception and Performance, 19(2), 331-351.

Trick, L. M., \& Pylyshyn, Z. W. (1994). Why are small and large numbers enumerated differently? A limited-capacity preattentive stage in vision. Psychological Review, 101(1), 80-102.

Watson, D. G., Maylor, E. A., \& Bruce, L. A. M. (2007). The role of eye movements in subitizing and counting. Journal of Experimental Psychology: Human Perception and Performance, 33, 1389-1399.

Wynn, K. (1997). Competence models of numerical development. Cognitive Development, 12, 333-339. 